Mots. Les langages du politique

Violence et démocratie en Amérique latine

\title{
La guerre des mots dans la transition mexicaine
}

\section{Hélène Combes}

\section{OpenEdition}

Journals

Édition électronique

URL : https://journals.openedition.org/mots/1172

DOI : $10.4000 /$ mots. 1172

ISSN : 1960-6001

\section{Éditeur}

ENS Éditions

\section{Édition imprimée}

Date de publication : 1 novembre 2007

Pagination : 51-64

ISBN : 978-2-84788-113-4

ISSN : 0243-6450

\section{Référence électronique}

Hélène Combes, «La guerre des mots dans la transition mexicaine », Mots. Les langages du politique [En ligne], 85 | 2007, mis en ligne le 01 novembre 2009, consulté le 24 avril 2022. URL : http:// journals.openedition.org/mots/1172; DOI : https://doi.org/10.4000/mots.1172

\section{(c) ENS Éditions}




\section{Hélène Combes}

\section{La guerre des mots dans la transition mexicaine}

Depuis 1988, le Mexique a connu la mise en place progressive d'un système tripartisan. Le Parti révolutionnaire institutionnel (PRI), héritier de la révolution mexicaine, né à la fin des années vingt, consolidé dans les années trente autour d'un régime semi-corporatiste, reste cependant au pouvoir jusqu'en 2000.

Au cours de cette longue période, le régime connait des phases d'ouverture et de fermeture, la palette du rapport aux groupes d'opposition allant de l'exclusion du jeu électoral à la répression physique en passant par la cooptation. Le Parti Action nationale (PAN), né en 1939 de groupes contre-révolutionnaires et de leur réaction à une reprise du «programme révolutionnaire», constitue jusqu'en 2000 le principal parti d'opposition. Il est le seul à ne pas être interdit ou à pouvoir remplir les critères pour participer à la vie électorale, le seul à avoir aussi eu une vie partisane continue. Clairement situé à droite, implanté historiquement dans le centre et le nord du pays, le PAN remporte l'élection présidentielle de 2000.

En 1989, un troisième parti fait son apparition: le Parti de la révolution démocratique (PRD). Il nait de la rencontre entre plusieurs petits partis de gauche, des mouvements sociaux, des « organisations sociales» (associations) nées de la cristallisation de ces derniers et le Courant démocratique (CD) du PRI. En 1988, lors des résultats de l'élection présidentielle, alors que le candidat du Front de gauche (Cárdenas) est en tête, le système informatique tombe en panne. Une fois celui-ci réparé, c'est le candidat du PRI, Carlos Salinas de Gortari, qui est donné vainqueur. La fraude - ou ce qui est vécu comme tel par les partisans de Cárdenas - provoque, par réaction, une résistance civique de grande envergure. Ce mouvement contribue à l'accélération du rassemblement de la gauche mexicaine apparu dès le milieu des années quatre-vingt. Il donne naissance au PRD.

Donc, à partir du début des années quatre-vingt-dix, c'est autour de ces trois partis que se développe la vie politique nationale et c'est aussi dans leurs interactions que se joue en grande partie la trajectoire de la transition démocratique mexicaine. Pour toute une série de raisons que nous allons évoquer au fil de cet article, le PRD occupe une place singulière, devenant l'« opposition » par

Université Paris 1, CNRS (CRPS), combeshvc@yahoo.com 
excellence. Une manière de comprendre le positionnement du PRD dans l'espace politique national est d'étudier le registre de disqualification ${ }^{1}$ mobilisé par ses adversaires. Le registre verbal utilisé constitue un marqueur de la place du PRD dans l'espace politique national et devient donc une manière de cerner la place de ce parti dans la transition mexicaine.

Pour ce faire, notre recherche lexicale s'est centrée sur quelques moments charnières de la vie politique nationale. Sur ces périodes charnières - 1991, 1994, 2003, 2006 - nous avons travaillé à partir d'un corpus de déclarations de dirigeants du PRI reprises par des sources journalistiques. Pourquoi ces dates sont-elles des périodes charnières? 1991 correspond aux élections de mimandat de Carlos Salinas de Gortari et constitue la période de plus forte offensive du régime contre l'opposition. 1994 est l'année de l'émergence de l'Armée zapatiste de libération nationale, de l'assassinat du candidat du PRI à la présidence et de l'élection présidentielle. 2003 correspond au début de l'entreprise de délégitimation des politiques du maire de Mexico, Andres Manuel López Obrador, et des débuts du lancement de la procédure de destitution. 2006 correspond à nouveau à une élection présidentielle particulièrement conflictuelle. Le corpus de textes provient principalement de deux journaux: le grand hebdomadaire politique Proceso et le quotidien d'opposition La Jornada. Dans le cas du PAN, et pour la période récente, nous avons aussi analysé les déclarations de dirigeants dans le journal interne du parti, La Nación. Le passage de la presse généraliste à la presse partisane pour l'étude de ce registre de disqualification s'explique essentiellement par un problème d'accès aux sources partisanes du PRI sur la première période. Ce passage introduit cependant sans doute un biais. Les lecteurs doivent avoir à l'esprit que, dans le cas du PAN, ces déclarations - même si elles sont rendues publiques via Internet - ne sont pas passées par le filtre de la déclaration publique.

\section{«Vote pour le candidat de la paix» : faire obstacle au «parti violent»}

«Dans le monde entier, les peuples qui ont écouté les incitations à la violence souffrent ensuite l'horreur de la guerre. Vote pour le candidat de la paix. $»^{2}$ Ce slogan de campagne du PRI dans un État fédéré, bastion du PRD - le Michoacán -, résume assez bien la bataille des mots qui fait rage au début des années quatre-vingt-dix pour qualifier l'opposition qui commence indéniablement à grignoter le monopole du PRI. Face aux accusations de «régime auto-

1. Par registre de disqualification, nous entendons l'ensemble des mécanismes oratoires tendant à décrédibiliser le PRD comme acteur à part entière du processus démocratique en cours.

2. Exemple de slogan du PRI. Campagne électorale dans l’État de Michoacán. 
ritaire», de «dictature», le PRI, par la voix de son président, se posait alors en garant de la stabilité politique et sociale :

[...] votez pour apporter la tranquillité dans vos foyers [car] il existe l’intention cachée dans certaines activités de créer un climat de terreur pour empêcher l'expression du vote. [Les partisans du PRD] sont en train de favoriser la violence et c'est ce que nous devons dénoncer ${ }^{3}$.

Dans la première moitié des années quatre-vingt-dix, alors qu'il connait ses premiers balbutiements de grand front de gauche, le PRD est présenté par le PRI comme un «parti violent » susceptible de mettre en cause la stabilité politique mexicaine :

Le PRD survit là où il y a de la violence [...]. On note la présence du PRD là où il a de la violence, là où il y a des confrontations, là où il y a des affrontements, là où il y a de l'anarchie et une situation d'insécurité pour la population ${ }^{4}$.

Ce registre de la disqualification prenait essentiellement racine dans le terreau des conflits postélectoraux qui se développaient dans la première moitié des années quatre-vingt-dix, comme en atteste cette déclaration du président de la République, Carlos Salinas de Gortari, devant des entrepreneurs :

Nous savons qui et ce qui déstabilise [...] le Michoacán [État du centre où se sont développés les principaux conflits postélectoraux au début des années quatrevingt-dix. NDA]. Nous ne permettrons pas des agressions et des intransigeances. Les conflits électoraux ne retarderont pas le développement de la région ${ }^{5}$.

Au Mexique, la régularité des scrutins a été - et reste aujourd'hui - au cœur de la démocratisation. Lutte pour la démocratie et lutte pour des élections libres et transparentes se confondent. Cela tient au rôle que n'ont cessé de jouer les irrégularités électorales dans ce système politique. La nature «frauduleuse » des élections caractérise le régime semi-autoritaire. Mettre fin au monopole du PRI comme parti officiel ne peut alors passer, pour l'opposition, que par la reconquête d'un vote libre et donc par la bataille « dans » et « autour » des urnes. C'est donc autour des stratégies de contestation des résultats que se noue la bataille d'attribution du label de «parti violent » (partido violento), label symptomatique de la place du PRD dans le système politique mexicain. Il convient par conséquent de s'arrêter sur ces conflits afin de mieux comprendre comment ces derniers ont structuré la place du PRD dans le système politique national, le discours du PRI étant à la fois un indice et un élément structurant de ce positionnement.

3. Entretien avec le président du PRI, Fernando Colosio, cité par Proceso, n 660-11, 26 juin 1989.

4. Intervention devant le Congrès législatif d'un député du PRI, cité par Proceso, $\mathrm{n}^{\circ} 701-703,9$ avril 1990.

5. Déclaration, fin février 1993, du président de la République, Carlos Salinas de Gortari, cité par Proceso, $\mathrm{n}^{\circ} 940-943,7$ novembre 1994. 


\section{Violence et conflits postélectoraux}

Quinze États sur trente-et-un, au cours des années quatre-vingt-dix, connaissent un conflit postélectoral de grande ampleur, si bien que certains auteurs ont parlé d' "élections interminables ${ }^{6}$. Dans quasiment tous les États de la République, on assiste à des contestations sporadiques des résultats électoraux. Todd Eisenstadt a réalisé un décompte précis de l'ensemble des conflits électoraux au Mexique entre 1989 et 2000 à partir d'un dépouillement de la presse locale et régionale (Eisenstadt, 2003). Il a recensé 936 conflits postélectoraux au cours de 8785 élections. D'après son travail, entre 1989 et $2000,10 \%$ des élections mexicaines ont donné lieu à un conflit postélectoral. $78 \%$ des conflits postélectoraux ont été l'œuvre du PRD et $22 \%$ celle du PAN. À partir de 1989 , le PRD devient donc le principal acteur de la lutte antifraudes. Or, au cours de ces mouvements postélectoraux, la répression est importante: 245 militants ou sympathisants du PRD meurent durant la période 1989-1994. Dans un travail sur la violence électorale, nous avons constaté que $64 \%$ de ces militants perredistes tués le sont dans un contexte électoral, $16 \%$ d'entre eux dans un contexte préélectoral ou le jour de l'élection et $84 \%$ dans un conflit postélectoral. Le répertoire du mouvement antifraudes est particulièrement sujet à la répression.

Tableau 1. Moment de l’homicide (1989-1994)

\begin{tabular}{|l|c|c|}
\hline & $\begin{array}{c}\text { Dans le cadre de leur } \\
\text { appartenance au mouvement } \\
\text { contre les fraudes } \\
(\mathbf{n = 2 4 5 )}\end{array}$ & $\begin{array}{c}\text { Dans le cadre d'une } \\
\text { opération de maintien } \\
\text { de l'ordre } \\
(\mathbf{n}=66)\end{array}$ \\
\hline $\begin{array}{l}\text { Durant ou à la sortie d'une } \\
\text { réunion partisane }\end{array}$ & $13 \%$ & - \\
\hline Pendant une manifestation & $9 \%$ & $32 \%$ \\
\hline $\begin{array}{l}\text { Pendant une occupation } \\
\text { de mairie }\end{array}$ & $8 \%$ & $30 \%$ \\
\hline Pendant un campement & $6 \%$ & $23 \%$ \\
\hline $\begin{array}{l}\text { Durant ou à la fin d'un } \\
\text { meeting électoral }\end{array}$ & $9 \%$ & - \\
\hline $\begin{array}{l}\text { Dans l'exercice d'une fonction } \\
\text { élective }\end{array}$ & $6 \%$ & - \\
\hline $\begin{array}{l}\text { Pendant une autre activité } \\
\text { militante }\end{array}$ & $33 \%$ & - \\
\hline Hors activité militante & $9 \%$ & - \\
\hline
\end{tabular}

Source : Hélène Combes, base de données sur la violence électorale entre 1989 et $1994^{7}$.

6. Enrique Krauze reprenant l'expression de Gabriel Zaid (Krauze, 1997, p. 430).

7. Base de données élaborée à partir de : Comisión nacional de derechos humanos, Informe de la comisión nacional de derechos humanos sobre las quejas presentadas por el PRD, Mexico, 
La manifestation est le répertoire d'action le plus fréquent. À côté des revendications dont elles sont porteuses, les manifestations représentent en ellesmêmes un défi aux autorités. Elles s'inscrivent dans une réappropriation forte de l'espace public. Si elles deviennent presque routinières au cours des années quatre-vingt-dix, elles n'en sont pas pour autant anodines. $14 \%$ des perredistes tués le sont durant une manifestation. Ce chiffre passe à $31 \%$ si l'on circonscrit l'analyse aux opérations de maintien de l'ordre.

La deuxième forme d'action faisant appel au nombre est le campement. Le campement protestataire se caractérise généralement par l'installation de tentes sur la place centrale de la ville, qui est, au Mexique, le lieu de concentration des pouvoirs exécutif, religieux et parfois législatif. Ce centre, aussi véritable cœur de la ville (commerces, loisirs, etc.), offre une grande visibilité à la contestation et constitue une arène propice à son expression. Choisir le Zócalo, c'est aussi symboliquement inscrire la protestation au cœur même du pouvoir national et le défier, ce qui débouche sur un échelon supplémentaire de pression de la part des autorités. Ainsi, le coût de l'engagement lors d'un campement est élevé. En effet, $23 \%$ des homicides commis dans le cadre d'une opération de maintien de l'ordre ont eu lieu lors d'un campement et $9 \%$ pour l'ensemble des homicides.

Troisième forme d'action : l'occupation des mairies. Face à ce qui est considéré comme des fraudes électorales, les mouvements postélectoraux tentent de rétablir le «résultat des urnes » de deux manières: par l'occupation de mairies et par la mise en place de municipes parallèles. L'occupation des mairies est un mode d'action relativement récurrent des mouvements antifraudes. D'ailleurs, Inglehart, dans une vaste étude comparatiste (Word Values Surveys), place le Mexique au deuxième rang des pays où des édifices institutionnels sont occupés (Inglehart, 1997).

Les États du Michoacán et du Guerrero ont connu en 1989-1990 les expériences d'occupation de mairies les plus spectaculaires. Dans le premier cas, 72 mairies sur les 113 de l'État ont été occupées. Cela marque un durcissement du conflit et correspond à un cran supplémentaire dans la radicalisation du répertoire d'action. De l'occupation symbolique du centre des pouvoirs que représente le campement au Zócalo, on passe à l'occupation effective du lieu du pouvoir. Le PRD met en place des conseils municipaux parallèles : 23 au Michoacán en 1989 (sur 113), 20 au Guerrero (sur 75), 7 à Puebla (sur 217). Ainsi, au Guerrero, les militants qui occupent les mairies exercent les fonctions administratives les plus ordinaires : célébration des mariages, gestion du cadastre et perception des impôts locaux. Dans certains endroits, les conseils

1994 ; Fundación Ovando y Gil y Secretaría de derechos humanos del PRD, Crónica de la violencia política. Guerrero, Mexico, octobre 1998, vol. 1 (189 p.) et vol. 2 (116 p.) ; (GP) : Grupo parlamentario del PRD, En defensa de los derechos humanos. Un sexenio de violencia política, PRD, 1994. 
municipaux s'installent chez des particuliers. Tout un réseau de solidarité se met en place pour assurer le maintien des institutions parallèles: donations financières, matérielles et alimentaires. Les cloches sont utilisées pour avertir de l'intervention de la police de l'État (Calderon Molgara, 1999, p. 229). Les occupations de mairies, encore moins tolérées que les campements, donnent souvent lieu à des expulsions violentes suivies d'incarcérations. Les occupations de mairies sont à l'origine de $8 \%$ des victimes de violence politique et de $30 \%$ des morts dans le cadre d'une opération de maintien de l'ordre. Elles ont été particulièrement réprimées dans les États de Oaxaca, du Michoacán et du Guerrero. La force symbolique des occupations de mairies mais aussi leurs implications matérielles ne sont pas étrangères à l'ampleur de la réaction.

\section{Les «émetteurs» de la violence}

Tableau 2. Responsables matériels présumés des homicides en période électorale

\begin{tabular}{|l|c|c|}
\hline Militants PRI & 48 & $31 \%$ \\
\hline Police (locale ou fédérale) & 42 & $27 \%$ \\
\hline Pistoleros & 33 & $21 \%$ \\
\hline Autorité municipale & 9 & $6 \%$ \\
\hline Autres dont l'armée & 22 & $14 \%$ \\
\hline Sans réponse & 1 & $1 \%$ \\
\hline
\end{tabular}

Source : Hélène Combes, base de données sur la violence électorale entre 1989 et 1994.

Qui sont les «émetteurs » de la violence ? La répression est polycéphale et multiforme. Les militants priistes seraient les premiers responsables matériels des homicides en période électorale (dans $31 \%$ des cas). Cependant, il est difficile de dissocier complètement militants du PRI, autorités locales, armée et police. Quand la violence est l'œuvre de militants priistes ou d'autorités locales, bien souvent la police vient prêter main-forte. La police - locale ou fédérale -, quant à elle, serait l'auteur de $27 \%$ des homicides (ce chiffre est de $37 \%$ dans l'État du Guerrero, ce qui la place comme principale responsable des homicides et est à mettre en relation avec son passé de lutte contre la guérilla dans les années soixante-dix). Viennent ensuite les pistoleros (hommes de main armés) à la solde de caciques ou de dirigeants du PRI. Les commanditaires (les «assassins intellectuels », selon l'expression mexicaine) seraient avant tout les autorités municipales (dans $37 \%$ des cas) suivies des dirigeants priistes (dans $27 \%$ des cas).

La violence et les homicides dans le cadre d'opérations de maintien de l'ordre varient aussi en fonction du répertoire d'action mobilisé par les acteurs. Les conflits postélectoraux montrent que la naissance d'une civilité électorale, à l'échelle locale, susceptible de déboucher sur la forclusion de la violence n'est 
pas parallèle à la transition démocratique nationale. L'«irruption des masses» dans la transition mexicaine à l'échelon local se traduit par une forte répression. Cette répression semble moins présente dans d'autres transitions latinoaméricaines qui se sont réalisées «par le haut», grâce à un pacte entre élites. Paradoxe de la situation mexicaine (Shatz, 2001) : un des régimes considéré comme le plus stable de la région dans les années soixante-dix donne lieu à l'une des transitions les plus violentes, mais cette violence de la part du régime est pourtant niée, occultée et non prise en compte par les analystes de la transition mexicaine, à de rares exceptions près (Cadena Roa, 2003).

Comment comprendre alors que le gouvernement du PRI ait ainsi imposé dans l'opinion publique l'image du PRD comme "parti violent », alors même que les faits attestent d'une répression importante à l'égard de ce dernier? Au-delà de la simple explication d'un certain contrôle des médias par le pouvoir, comment la thèse du «parti violent» a-t-elle été relayée de manière plus ou moins explicite par des analystes, politologues et autres chroniqueurs? Pourquoi le PRI a-t-il gagné cette bataille des mots qui fait que, comme nous le verrons, ce label est encore utilisé quinze ans après?

C'est bien autour de ces conflits électoraux que se sont noués l'usage et l'enracinement de ce qualificatif pour le PRD, raisons pour lesquelles il nous semblait essentiel de revenir en détail sur ces derniers. Notre première hypothèse est que le recours à la mobilisation apparait comme un mode illégitime et donc se prêtant à être disqualifié, car il ne correspond pas au canon de la «bonne» transition définie par les pères fondateurs de la «transitologie» et repris à leur compte par les élites «démocratisantes».

En effet, pour Adam Przeworski, si les mouvements sociaux persistent après la phase de libéralisation, ils risquent fort de déstabiliser les élites civiles et militaires (Przeworski, 1991, p. 56). «Si la mobilisation des opposants au régime paraît aller "trop loin", le régime autoritaire peut de nouveau se sentir indispensable» (O’Donnell, Schmitter, Whitehead, 1986, p. 49). Donc, pour les transitologues, dans les années quatre-vingt et au début des années quatre-vingt-dix, il est souhaitable que le rôle des mouvements sociaux soit limité car il pourrait être néfaste. En effet, les mobilisations populaires seraient dangereuses. D'ailleurs, le vocabulaire utilisé pour décrire les mobilisations par les transitologues, aussi bien quand elles sont porteuses d'ouverture que lorsqu'elles deviennent indésirables, est symptomatique : couches explosives de la société, indocile secteur populaire, acteur ambigu, théâtre de rue, etc. En fait, les transitologues semblent largement s'être ralliés à l'opinion des élites de certains pays avec lesquelles ils ont d'ailleurs, dans de nombreux cas, tissé des liens très étroits (d'amitié ou d'échange d'expertise). Ces élites voient dans les mouvements sociaux un risque d'instabilité politique pouvant entrainer le retour des duros (des durs du régime) et aussi une remise en cause de leur manière de mener à bien les transitions. Ainsi, Terry Karl insiste sur le fait que pour le 
succès des transitions, le consensus et les compromis sont nécessaires (Karl, 1990, p. 17). Par conséquent, la démobilisation serait une nécessité pour la pérennité des démocraties renaissantes. En résumé, pour les transitologues, les mobilisations populaires influenceraient le processus démocratique dans la mesure où elles incitent le régime à l'ouverture. Elles rendent plus facile la rupture des modérés d'avec les duros. Le PRD a donc eu recours à une stratégie perçue comme illégitime par les analystes qui diffusent, dans le Mexique des années quatre-vingt-dix, une vulgate de la transitologie. Il usait d'une stratégie qui était perçue comme pouvant mettre à mal le déroulement de la «bonne transition ». Le gouvernement, de son côté, aurait fait un travail de maintien de l'ordre, «pour la paix sociale» - autre slogan du PRI en 1991.

Au-delà de cela, le label de "parti violent» s'est imposé d'autant plus facilement que quelques dirigeants du PRD viennent d'anciennes guérillas et qu'un tiers sont des leaders ou anciens leaders de mouvements sociaux. Le temps continental favorisait la stigmatisation de toute filiation à une guérilla, alors même que la période, notamment en Amérique centrale, était au désarmement. Cet usage du parallèle entre PRD, guérilla et violence (et plus largement mouvements sociaux et violence) est réactivé en 1994 au moment où surgit l'Armée zapatiste de libération nationale (EZLN). En effet, le candidat du PRD à la présidentielle, Cuauhtémoc Cárdenas, se rapproche durant la campagne du sous-commandant Marcos. L'EZLN n'a pas encore l'aura internationale dont elle bénéficiera quelques années plus tard et n'est pas encore présentée comme la première guérilla post-guerre froide. Le PRI diffuse alors des affiches de campagne montrant Cárdenas et Marcos côte à côte avec le slogan : «Le candidat de la violence ».

Cette thématique du choix de la paix contre celui du «parti de la violence» est récurrente dans la première moitié des années quatre-vingt-dix. Elle est présente à chaque échéance électorale, les législatives de 1991 et surtout la présidentielle de 1994. Donc, dans la première moitié des années quatre-vingt-dix se structure un champ de la qualification qui perdure ensuite, signe du positionnement du PRD dans le système politique, entre inclusion et exclusion. On retrouve les accusations de "parti violent» au cours des années 2000 dans des conjonctures bien spécifiques, et ce malgré une évolution du profil public du PRD (notamment de par la victoire de gouverneurs dans des États fédérés). Cependant, au cours des années 2000 , le registre de disqualification se diversifie, diversification aussi bien liée aux évolutions du système politique national qu'au temps continental. 


\section{De «violent » à « populiste »}

Le Mexique connait en 2000 une alternance historique. Le PAN remporte l'élection présidentielle. Parallèlement, le PRD confirme son implantation à Mexico, en gagnant la mairie pour un deuxième mandat. On assiste pendant cette période à un glissement des modes de disqualification et au basculement de l'appellation de «parti violent» à celle de «populiste». Ce glissement s'opère autour de la figure du maire de Mexico, Andrés Manuel López Obrador. Plusieurs raisons peuvent être avancées pour comprendre ce phénomène. Ce glissement s'explique en premier lieu par la fin de l'affrontement entre ce que l'on nomma le PRI-État et le PRD dans le contexte des conflits postélectoraux. C'est désormais sur le terrain de son exercice de gouvernement local que le PRD est jaugé. Cette tendance est renforcée par l'arrivée du PAN au pouvoir. Non seulement les relations du PAN et du PRD ne sont pas inscrites, avant les élections présidentielles de 2006, dans le cadre des affrontements postélectoraux mais, de plus, le PAN met l'accent sur la «bonne gouvernance » et la « gestion responsable des finances publiques». Enfin, dernière raison liée cette fois plus au temps continental, le spectre du populisme incarné par le Vénézuélien Hugo Chávez fait son apparition dans le paysage idéologique.

\section{Le spectre de Chávez}

«Ne votez pas pour le populisme. [...] Tournez le dos "à ce monsieur de Mexico" ${ }^{8}$ : telle est l'exhortation faite aux Mexicains par Mario Vargas Llosa, le grand écrivain latino-américain et ancien candidat présidentiel conservateur au Pérou. Et de réitérer ces conseils : "Je vous demande de ne pas voter pour un populiste. [...] Choisissons bien. Qu'il ne nous arrive pas la même chose qu'à nos pauvres frères vénézuéliens " ${ }^{9}$, dit-il, faisant tout aussi bien référence à la situation péruvienne que mexicaine. L'entrée en scène de Vargas Llosa intervient relativement tard dans le débat sur le «populisme» supposé d'Obrador. Il ne vient en cela, comme nous le verrons, qu'ajouter sa plume à un espace de polémique déjà structuré en ces termes. Mais son intervention est marquante à plusieurs titres. Car, même s'il n'est sans doute pas un fin connaisseur de la vie politique mexicaine, sa formule «la dictature parfaite » pour qualifier le régime du PRI fit florès et fut mobilisée par les analystes du régime et les adversaires de tout poil. Sa prise de position montre à quel point on se situe dans un espacetemps régional.

8. Discours prononcé lors de la réception d'un doctorat honoris causa de l'université de San Luis Potosí.

9. Discours au salon du livre de Guadalajara, le plus grand salon du livre d'Amérique latine, fin novembre 2005 . 
Retour au début de la polémique. Dès 2003, Obrador est présenté par nombre de ces opposants comme un Hugo Chávez mexicain. Son style politique, ses appels au peuple et aussi ses politiques sociales sont comparés à ceux du président vénézuélien. Jorge Castañeda, ancien ministre des Affaires étrangères, déclare qu'il faut se débarrasser d'Obrador par tous les moyens («las buenas o las malas»). Dans une stratégie d'évincement, faire échec à Obrador, incarnation du risque "populiste» au Mexique, semble justifier tous les moyens utilisés, et cela même si l'État de droit est remis en cause. Ainsi, par exemple, le PRI et le PAN font alliance et votent la levée de l'immunité du maire de Mexico, sur la base d'une affaire mineure d'interdiction de construction d'une voie d'accès à un hôpital. Le lancement de cette procédure implique l'impossibilité d'être candidat à la présidentielle, si bien que certains analystes politiques ont pu parler d'une dérive vers un «coup d'État préventif». Un mouvement civique de grande ampleur s'est alors déclenché. Des comités de soutien ont été créés dans tout le pays, notamment à travers la réactivation et la reconversion des réseaux liés à la lutte contre les fraudes électorales. Le 24 avril 2005, un million de personnes participent à une manifestation à Mexico. Dans un climat quasi insurrectionnel, Obrador lance un appel au calme. Le procureur de la justice est acculé à la démission et le président Vicente Fox demande personnellement que la procédure soit suspendue. Obrador appelle à la démobilisation. S'instaure alors un fragile équilibre caractérisé cependant par une radicalisation des deux camps, qui a marqué de son empreinte la campagne électorale des présidentielles de 2006. Le responsable de la formation politique au sein du Comité exécutif du PAN n'hésite pas, par exemple, à publier un appel à la mobilisation contre Obrador en ces termes :

Du cas de Hitler, qui est déjà passé à la postérité et du cas de Chávez, qui est en train d'avoir lieu, il y a une leçon de première importance pour les nouvelles générations $[. .$.$] : réfléchis et mets à profit ton vote, sois prudent à l'heure de choisir car l'autorité$ que tu élis, les jours passant et obnubilé par le pouvoir, peut devenir ton assassin ou celui de ta propre famille ${ }^{10}$.

Plus généralement, c'est donc au nom de la lutte continentale contre le «populisme » que cette mobilisation est justifiée, ce qui renvoie à un registre de disqualification qui dépasse largement l'échelle nationale. Cette lutte contre le populisme se fonde aussi sur la division entre une «bonne » et une « mauvaise » gauche latino-américaine. Un économiste du PAN, ancien député, déclare par exemple :

Sur les bases de ses propres déclarations et programmes, le projet de nation du PRD se situe plus près de celui de Chávez au Venezuela que des gouvernements chiliens

10. Article de Javier Brown César dans La Nación, le journal interne du PAN, 30 mai 2006. Dans La Nación, cet exemple de parallèle entre Hitler et Obrador n'est pas isolé. 
qui sont ceux qui ont eu du succès. [...] [Dans le cas chilien], en arrivant au pouvoir après Pinochet, la gauche a adopté une position intelligente et moderne. Bien qu'elle ait accusé le général de violation des droits de l'homme, elle a maintenu et a renforcé beaucoup de changements initiés pendant cette période : privatisation, ouverture, réformes des pensions, réformes fiscales et du droit du travail ${ }^{11}$.

Donc, c'est au nom de la lutte contre une gauche «populiste » et « dangereuse » que des secteurs du PAN justifient aussi bien des modes d'action questionnant l'État de droit - la procédure de destitution sur des critères douteux ${ }^{12}$ que la violence de son registre de lutte politique. La référence fréquente au contexte vénézuélien, et plus ponctuellement à l'Allemagne de 1933, n'est pas anodine. À contexte exceptionnel, réponse exceptionnelle, semblent dire certains panistes. Au-delà du cadre général d'un discours contre l'avènement d'un régime "populiste», le discours du PAN prend racine dans l'analyse économique. Resserrons désormais notre analyse sur l'argumentaire de l'appareil partisan du PAN ${ }^{13}$.

\section{«Populisme» versus «gestion publique responsable»}

Le PAN place cette lutte contre le populisme essentiellement sur le terrain économique. Donc, les panistes dans leurs tribunes mettent surtout en avant une "gestion publique responsable» contre le "populisme» supposé d’Obrador. Le candidat [et désormais président de la République (2006-2012)] Felipe Calderón déclare ainsi : «Les finances du pays ne vont pas supporter un gouvernement populiste. $»^{14}$ Pour un autre paniste, Federico Dring Casar, «la version tabasceña [Tabasco, État d'origine d'Obrador] de Hugo Chávez non seulement représente une crise économique assurée, mais de plus porte le risque d'une profonde crise sociale. $»^{15}$

Deux actions gouvernementales locales sont au cœur des accusations des panistes visant la gestion irresponsable et populiste de la part d'Obrador. La première est la construction d'un deuxième niveau au périphérique de Mexico. Travail pharaonique, il est présenté comme un gouffre financier et un projet tape-à-l'œil. La deuxième mesure, qui présente un plus grand intérêt, est la création d'une retraite universelle pour les personnes âgées. Toute personne de plus de soixante-dix ans, quel que soit son revenu, peut demander une «bourse pour personne âgée ». Au Mexique, les retraites modiques permettent rarement

11. Luis Pazos, El Financiero, 31 mai 2006.

12. Des juristes fort éloignés du PRD et s'étant intéressés au fond de l'affaire ont souligné le caractère absurde - d'un point de vue juridique - de la démarche engagée contre Obrador. Voir Roldán Xopa, 2004.

13. Nous utilisons ici un corpus plus réduit basé sur la production de textes de dirigeants du PAN dans des quotidiens nationaux ou dans le journal interne du PAN.

14. www.jornada.unam.mx/2006/03/18/oogn1pol.php

15. Article de Federico Dring Casar dans La Nación, le journal interne du PAN, 6 juillet 2006. 
aux personnes âgées de vivre sans un appui de leur famille ou une activité professionnelle complémentaire. Ce programme a essentiellement été développé dans les arrondissements les plus défavorisés de la ville et bénéficierait à 352000 personnes via l'attribution de la moitié d'un salaire minimum.

Politique sociale et redistributive modeste vue depuis l'Europe, cette pension fut taxée par les panistes d' "irresponsabilité politique» en raison de son coût, ainsi que de «pratique populiste et clientéliste». Dans le cadre de la campagne électorale, elle a donné lieu à une passe d'armes. Le PAN a lancé une campagne télévisuelle contre Obrador, l'accusant d'avoir augmenté la dette de Mexico et d'être porteur d'un projet économique populiste. Si bien que face aux attaques, ce dernier a même refusé de participer au premier débat présidentiel et, à travers le témoignage d'une écrivaine reconnue, se défend d'entretenir des liens avec Chávez :

On a parlé de populisme pour dénigrer, pour écraser, pour écarter Obrador. D’abord, voyons ce qu'est le populisme. C'est aider les personnes âgées grâce à une pension au lieu de voler l'argent, construire un deuxième étage [au périphérique] et remplir ses engagements ${ }^{16}$ ?

À ces arguments, les panistes rétorquent en invoquant le poids qu'une telle mesure impliquerait pour les finances publiques :

[...] La pension pour les personnes âgées qu'offre le candidat du soleil aztèque [emblème du PRD] s'élèverait à 43 millions de pesos par an. [...] Autrement dit, le pays serait en route vers une nouvelle dette comme celle qu'il a dû supporter avec un autre Lópe $Z^{17}$ et la faillite que nous avons vécue en $1982^{18}$.

Le même discours fut repris parCalderón lors de son meeting de fin de campagne à Mexico en juin 2006 et fut un des thèmes de campagne récurrents du PAN.

\section{Mélange des registres autour de l'élection de 2006}

La mobilisation contre la destitution, et surtout le climat de la campagne électorale de 2006, suite à une montée progressive des tensions entre le PAN et le PRD depuis 2000, a été un contexte favorable de réappropriation par le PAN des registres de disqualification utilisés auparavant par le PRI à l'égard du PRD. Ainsi, durant la campagne, le PAN a diffusé un spot télévisé, lui aussi finalement interdit par l'Institut fédéral électoral (IFE), intitulé «López Obrador est un danger pour Mexico ». Dans ce spot, on voyait Obrador en 1994, lors d'un

16. www.jornada.unam.mx/2005/11/29/ao4n2cul.php

17. En 1982, le Mexique connait, à la fin du mandat du président López Portillo, une crise économique sans précédent.

18. Article d'Ivan Paoli Bolio, directeur adjoint de la Fundación Adolfo Christlieb Ibarrola del PAN DF, La Nación, 15 mai 2006. 
conflit postélectoral dans son État d'origine - le Tabasco -, occuper des champs pétroliers. Apparaissaient aussi les images du lynchage de policiers par des habitants d'un quartier de Mexico alors qu'il était maire. De son côté, le PRD tentait de donner l'image d'un Felipe Calderón vendu aux intérêts des grands entrepreneurs.

Le contexte de l'élection du 2 juillet 2006 favorise d'autant plus la remobilisation de ce registre de «parti violent». Cette élection s'est caractérisée par un résultat électoral extrêmement serré entre López Obrador et Felipe Calderón. Après un nouveau décompte des procès verbaux, l'avantage de Calderón sur son adversaire est de $0,5 \%$ seulement. S'ensuivront deux mois de procédures contentieuses et de mobilisations de la part du PRD, avant que le Tribunal fédéral électoral tranche - le 6 septembre 2006 - en faveur de Calderón.

Au cours de ces deux mois, l'opposition énumère les multiples contradictions, incohérences, points obscurs du scrutin: des urnes perdues et retrouvées dans des poubelles, des pourcentages de plus de $80 \%$ pour le PAN dans les bureaux de vote où ni le PRI ni le PRD n'avaient de scrutateurs. C'est aussi tout le vieux répertoire de la fraude qui semble avoir été utilisé localement dans certains lieux de la République : le bourrage des urnes, la "souris folle» (technique qui consiste à inscrire les électeurs dans des bureaux de vote éloignés de chez eux et à les renvoyer de bureau de vote en bureau de vote), etc. Dans certains endroits, les réseaux corporatistes, avec la fin du parti-État, se sont ralliés au PAN au pouvoir. D'ailleurs, la leader du syndicat officiel des enseignants, syndicat anciennement pilier du système corporatiste du PRI, qui a appuyé la fondation d'un nouveau parti - lequel, dès la campagne, a fait allégeance au PAN -, est soupçonnée d'être la grande ordonnatrice de la fraude. La réalité est sans doute bien plus complexe et contrastée, résultat des fraudes locales (pas uniquement dans les rangs du PAN) et de la mauvaise organisation de l'élection de la part d'un IFE politisé et peu enclin à régler des dispositifs techniques, mais aussi rétif à financer ce qui avait fait en partie le succès de l'élection de 2000 : la formation des présidents de bureaux de vote. Un campement sur la place centrale de Mexico - le Zócalo - et sur une des avenues emblématiques de la capitale commence alors. Est remis au goût du jour un répertoire de la lutte antifraude qui n'est en rien nouveau et qui fut très largement mobilisé par le PRD à l'échelle régionale au cours des années quatre-vingt-dix. Outre les manifestations, les campements, le PRD empêcha, le $1^{\text {er }}$ septembre, le président de la République de lire le traditionnel message à la nation. Autant d'éléments qui font resurgir le label de «parti violent».

Les labels de disqualification du PRD sont un indice de la difficile insertion du PRD dans le système politique mexicain, et cela bien qu'il soit, depuis le début des années quatre-vingt-dix, le deuxième ou troisième parti national en 
fonction des élections. Dans la première phase de la transition, le PRD a opté pour la mobilisation plutôt que pour la négociation, contrairement à l'autre parti d'opposition, le PAN, ce qui a contribué à son label de «parti violent». Dans un deuxième temps, alors que l'insertion du PRD dans le système politique semble assurée, via des victoires électorales et la gestion de plusieurs États fédérés, la fragilité de son insertion se fait jour dans les accusations de «populisme », liées en grande partie à un espace-temps continental et au spectre de Chávez.

\section{Références}

CADENA RoA Jorge, 2003, "State pacts, elites and social movements in Mexico's transition to democracy», States, Parties and Social Movements, J. Golstone éd., Cambridge, Cambridge University Press, p. 107-146.

CAlderón Molgara Marco Antonio, 1999, Violencia política y elecciones municipales en Michoacán y Guerrero, Mexico, Maestría en sociología, Instituto de investigaciones Dr. José Maria Luis Mora, p. 229.

EISENSTADT ToddA., 2003, "Thinking outside the (ballot) boxe: informal electoral institutions and Mexico's political opening», Latin American Politics and Society, vol. $45, \mathrm{n}^{\circ} 1$.

INGLEHART Ronald, 1997, Modernization and Postmodernization. Cultural, Economical and Political Change in 43 Societies, Princeton, Princeton University Press.

KARL Terry L., 1990, "Dilemmas of democratization in Latin America», Comparative Politics, vol. 23, $\mathrm{n}^{\circ} 1$.

KraUze Enrique, 1997, La presidencia imperial (1940-1996), Mexico, Tusquets Editores.

O’Donnell Guillermo, SCHMitTer Philippe, WhITEHEAD Laurence, 1986, Transitions from Authoritarian Rule. Tentative Conclusions about Uncertain Transitions, Baltimore, Johns Hopkins University Press.

PRZEWORSKI Adam, 1991, Democracy and the Market, Cambridge, Cambridge University Press.

RoLdÁn Xopa José, 2004, Le desafuero de Andrés Manuel López Obrador ¿Que es la legalidad?, Mexico, Huber.

Shatz Sara, 2001, "A difficult birth: dissent, opposition and murder in the rise of Mexico's Partido de la revolución democrática (PRD) », Political Opportunities, Social Movements and Democratization, $\mathrm{n}^{\circ} 23$. 